\title{
Local Binary Patterns and Its Variants for Finger Knuckle Print Recognition in Multi-Resolution Domain
}

\author{
D. R. Arun1, C. Christopher Columbus ${ }^{2}$, K. Meena ${ }^{3}$ \\ ${ }^{1}$ Department of Information Technology, PSN College of Engineering and Technology, Tirunelveli, India \\ ${ }^{2}$ Department of Electrical and Electronics Engineering, PSN College of Engineering and Technology, \\ Tirunelveli, India \\ ${ }^{3}$ Department of Computer Science and Engineering, Veltech DR. RR \& DR. SR Technical University, \\ Chennai, India \\ Email: arundr@psncet.ac.in, columbus@psncet.ac.in, meen.nandhu@gmail.com
}

Received 6 May 2016; accepted 18 May 2016; published 22 August 2016

Copyright (C) 2016 by authors and Scientific Research Publishing Inc.

This work is licensed under the Creative Commons Attribution International License (CC BY).

http://creativecommons.org/licenses/by/4.0/

(c) (i) Open Access

\begin{abstract}
Finger Knuckle Print biometric plays a vital role in establishing security for real-time environments. The success of human authentication depends on high speed and accuracy. This paper proposed an integrated approach of personal authentication using texture based Finger Knuckle Print (FKP) recognition in multiresolution domain. FKP images are rich in texture patterns. Recently, many texture patterns are proposed for biometric feature extraction. Hence, it is essential to review whether Local Binary Patterns or its variants perform well for FKP recognition. In this paper, Local Directional Pattern (LDP), Local Derivative Ternary Pattern (LDTP) and Local Texture Description Framework based Modified Local Directional Pattern (LTDF_MLDN) based feature extraction in multiresolution domain are experimented with Nearest Neighbor and Extreme Learning Machine (ELM) Classifier for FKP recognition. Experiments were conducted on PolYU database. The result shows that LDTP in Contourlet domain achieves a promising performance. It also proves that Soft classifier performs better than the hard classifier.
\end{abstract}

\section{Keywords}

Biometrics, Finger Knuckle Print, Contourlet Transform, Local Binary Pattern (LBP), Local Directional Pattern (LDP), Local Derivative Ternary Pattern (LDTP), Local Texture Description Framework Based Modified Local Directional Pattern (LTDF_MLDN), Nearest Neighbor (NN) Classifier, Extreme Learning Machine (ELM) Classifier 


\section{Introduction}

Hand based biometric identification is a booming research area due to its low cost in acquiring data, its reliability in identifying individuals and its degree of acceptance by the user [1]. Most popularly used hand biometric traits are hand geometry, finger print, palm print, hand vein patterns, finger knuckle print and palm side finger knuckle print [2]. Among these, finger knuckle print is a newly emerged biometric trait in recent years. The texture in the outer finger surface, also called dorsum of hand, has the potential to do personal authentication. The texture design obtained by bending the finger knuckle of a person is unique and that can be used as a biometric trait. The skin pattern on the finger-knuckle is highly rich in texture due to skin folds and creases. Hence, recently proposed local binary pattern based descriptors such as LDN, LDTP and LTDP_MLDN are used to extract features from FKP images. FKP is more advantageous because of easy accessibility and contact-less image acquisition. Figure 1 shows the sample FKP image used in this paper.

Local orientation information captured by using Gabor filter is used for FKP recognition [3]. Zhang et al. proposed a new technique which comprises of orientation and magnitude features [4]. In later years, the combinations of local and global features are used for FKP recognition. Aoyama et al. proposed that Band Limited Phase-Only Correlation (BLPOC) method was adopted to match finger knuckle print [5]. Morales proposed FKP authentication based on Scale Invariant Feature Transform (SIFT) algorithm [6]. In order to improve the recognition accuracy, the combination of SIFT and SURF algorithms are used for FKP [7]. Adaptive Steerable Orientation Coding (ASOC) was introduced by Li et al. [8]. Riesz transform based FKP recognition is also experimented for achieving better results.

Multi-resolution methods provide powerful signal analysis tools, which are widely used in feature extraction, recognition, denoising, compression, detection and image retrieval applications. These techniques are used by Ekenel and Sankur [9] to reduce the loss in FKP recognition. Hence, a new combination of Local Binary Pattern derivatives in Contourlet domain is evaluated in this paper.

\subsection{Literature Survey}

FKP recognition may depreciate due to the images with poor illumination, noise and pose variations. When textural feature extraction techniques are applied as it is in images, it is expected that there will be great variation in the textural features due to these extrinsic factors. Texture features can characterize regularity, randomness, directionality and coarseness properties of patterns. FKP image thus can be viewed as a texture pattern exhibiting symmetry and regularity. Hence, texture can play a predominant role in FKP recognition. LBP was originally designed by Ojala et al. (2002) for texture description, but later extended to face recognition. Recently, many more texture models such as LDN, LDTP, and LTDF_MLDN are proposed and successfully applied for pattern recognition problem.

The recognition rate mainly depends on textural features captures from the FKP image. But these textural features are severely affected by external factors. Hence, multi-resolution techniques are applied to diminish this effect. Motivated and justified by this, the combination of multi-resolution technique and texture descriptors are exploited for FKP recognition to enhance the classification accuracy.

\subsection{Outline of the Proposed Approach}

This paper proposes a novel personal recognition system using Finger Knuckle Print. Figure 2 shows the block diagram of the proposed personal recognition system. Initially, the captured FKP images are preprocessed

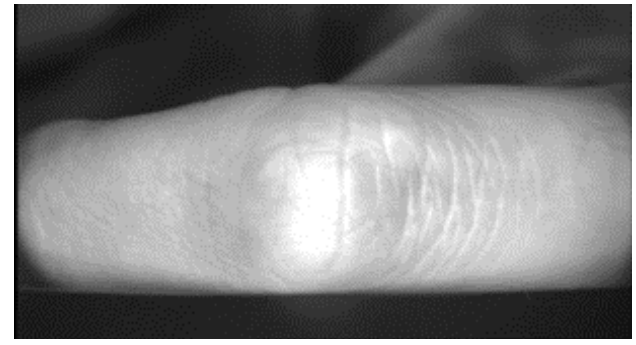

Figure 1. Sample finger knuckle print image. 


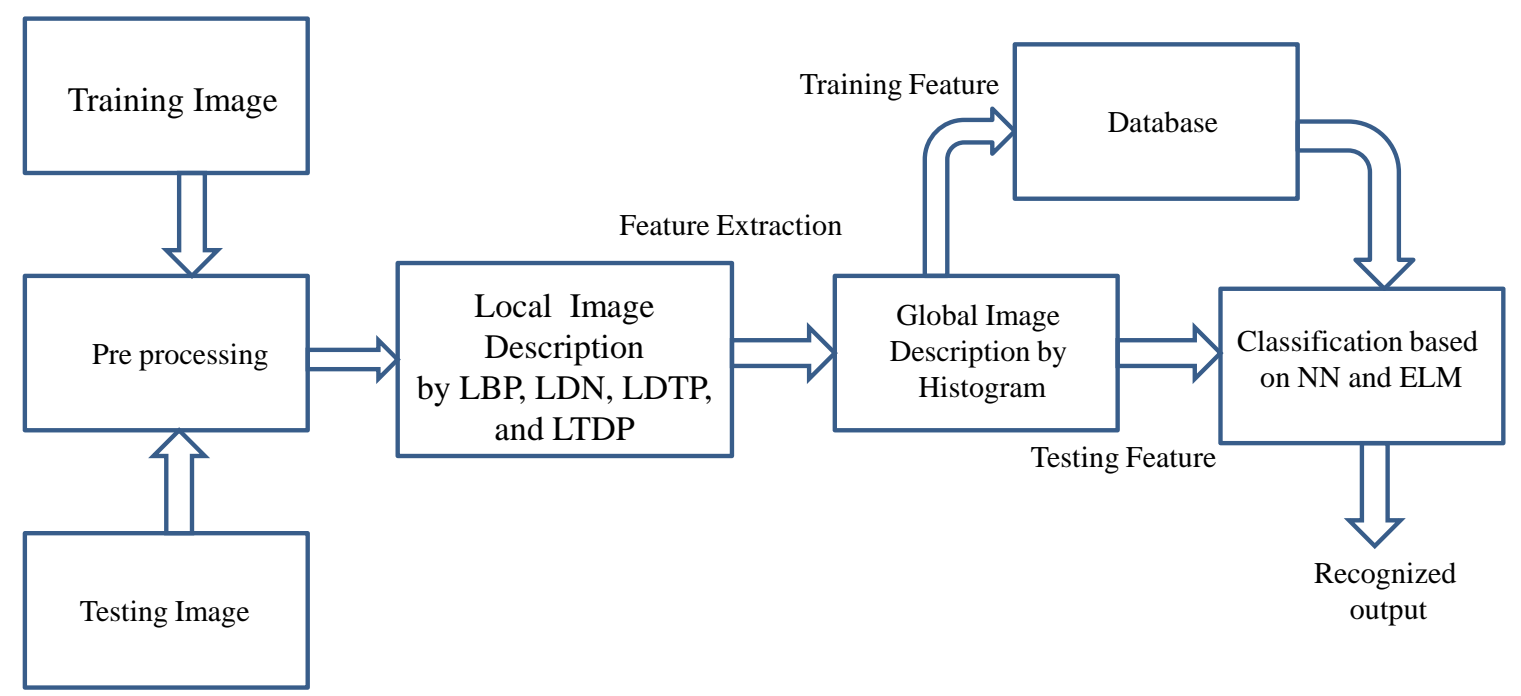

Figure 2. Block diagram of FKP recognition process.

by Contourlet transform which could handle FKP images with scaling, translation and rotation variant properties. This preprocessing approach also overcomes the problems that could arise due to distorted or impaired knuckle images. Secondly, the extracted ROI image of finger knuckle print is subjected to textural analyses for simultaneous extraction of textural feature information. The extracted feature vectors derived through the above methods are passed as input to the database. The texture feature of the testing images are computed and compared against the training features stored in the database using K-Nearest Neighbor and ELM classifier. Finally, the identity of a person is decided based on the matching scores derived from two different classifiers.

\subsection{Organisation of the Paper}

The rest of the paper is organized as follows: Section 2 describes the multi-resolution based preprocessing techniques in detail. Section 3 explains the various LBP based feature extraction techniques. Section 4 presents experimental results. Section 5 presents the conclusions and future enhancement of the proposed technique.

\section{Multi-Resolution Technique}

The main aim of the multi-resolution analysis is firstly, to obtain multiple evidences from the same image and search for those components that are less sensitive to intrinsic deformations due to extrinsic factors like illumination and noise. DWT, ridgelet, curvelet and Contourlet techniques are already experimented by the researchers for face recognition problem and the results shows that Contour let based preprocessing gives better results than the other techniques considered [10]. Multiresolution techniques extract both multi-scale and multi-directivity geometrical structures of the images. These geometrical structures are important to capture the intrinsic features such as lines and curves of a FKP images. Hence, Contourlet based preprocessing technique is used in this paper [11]. This method is a new image representation scheme which owns a powerful ability to efficiently capture the smooth contours of images. Contourlet transform can be divided into two main steps as Laplacian Pyramid (LP) decomposing and Directional Filter Banks (DFB). The original image is decomposed to a low pass image and a band pass image by LP decomposing. Each bandpass image is further decomposed by DFB. Repeating the same steps upon the low pass image, the multi-scale and multi direction decomposition of the image is obtained.

\section{LBP Based Feature Extraction}

\subsection{Local Binary Pattern}

LBP is computationally simple yet very efficient local texture operator [12]. These features are invariant to monotonic gray scale changes. LBP value of a sample $3 \times 3$ image is deliberated by, 


$$
\mathrm{LBP}_{p, r}=\sum_{p=0}^{p-1} s\left(g_{i}-g_{c}\right) 2^{i}, \quad S(x)= \begin{cases}1 & \text { if } x \geq 0 \\ 0 & \text { otherwise }\end{cases}
$$

where $g_{c}$ is the grey level value of the central pixel, $g_{p}$ is the grey value of its neighbours around $g_{c}$ and $P$ is the number of neighbours. A pattern number is computed by comparing the $g_{c}$ value with those of its neighbourhood. Figure 3 illustrates the basic LBP operator.

Conventional LBP requires 256 bins to store all possible patterns. The concept of uniform patterns is introduced to reduce the number of possible bins. It effectively captures the fundamental information of textures.

\subsection{Local Directional Number Pattern}

Adin Ramirez Rivera et al. introduced this descriptor to describe the structure and intensity transition of a texture pattern around a pixel in an image. The patterns are created with the edge response of the neighborhoods obtained by a compass mask and with the direction of the most positive and the most negative responses. The positive and negative responses are useful to determine the direction of the prominent bright and dark areas in the neighborhood. Two different compass masks such as kirsch and derivative-gaussian are experimented with LDN.

LDN assigns a six bit binary code to each pixel of an input image that represents the structure of the texture and its intensity transitions. Consequently, this pattern computes the edge response of the neighborhood using a compass mask and by taking the top directional numbers. The most positive and negative directions of those edge responses are used to describe LDN code. The more detailed explanation about LDN feature extraction is given in [13].

\subsection{Local Derivative Ternary Pattern}

Meena et al. proposed a new local texture descriptor called LDTP for face recognition under real time challenges. In LDTP, the directional features from Local Derivative Pattern (LDP) and three level descriptions from Local Texture Pattern (LTP) are fused with adaptive threshold technique (T). Figure 4 and Figure 5 represent the LDTP feature extraction in detail.

The more detailed explanation about LDTP feature extraction is given in [14].

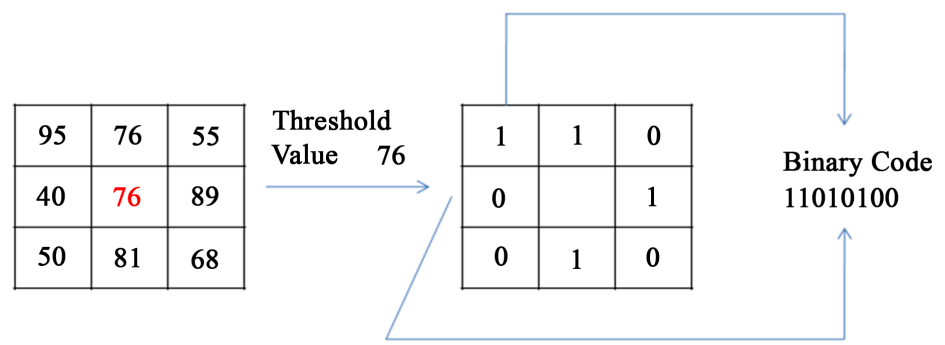

\section{Figure 3. Calculation of LBP.}

\begin{tabular}{|l|l|l|l|l|}
\hline $\mathrm{I}\left(\mathrm{Z}_{20}\right)$ & $\mathrm{I}\left(\mathrm{Z}_{21}\right)$ & $\mathrm{I}\left(\mathrm{Z}_{22}\right)$ & $\mathrm{I}\left(\mathrm{Z}_{23}\right)$ & $\mathrm{I}\left(\mathrm{Z}_{24}\right)$ \\
\hline $\mathrm{I}\left(\mathrm{Z}_{19}\right)$ & $\mathrm{I}\left(\mathrm{Z}_{1}\right)$ & $\mathrm{I}\left(\mathrm{Z}_{2}\right)$ & $\mathrm{I}\left(\mathrm{Z}_{3}\right)$ & $\mathrm{I}\left(\mathrm{Z}_{9}\right)$ \\
\hline $\mathrm{I}\left(\mathrm{Z}_{18}\right)$ & $\mathrm{I}\left(\mathrm{Z}_{8}\right)$ & $\mathrm{I}\left(\mathrm{Z}_{0}\right)$ & $\mathrm{I}\left(\mathrm{Z}_{4}\right)$ & $\mathrm{I}\left(\mathrm{Z}_{10}\right)$ \\
\hline $\mathrm{I}\left(\mathrm{Z}_{17}\right)$ & $\mathrm{I}\left(\mathrm{Z}_{7}\right)$ & $\mathrm{I}\left(\mathrm{Z}_{6}\right)$ & $\mathrm{I}\left(\mathrm{Z}_{5}\right)$ & $\mathrm{I}\left(\mathrm{Z}_{11}\right)$ \\
\hline $\mathrm{I}\left(\mathrm{Z}_{16}\right)$ & $\mathrm{I}\left(\mathrm{Z}_{15}\right)$ & $\mathrm{I}\left(\mathrm{Z}_{14}\right)$ & $\mathrm{I}\left(\mathrm{Z}_{13}\right)$ & $\mathrm{I}\left(\mathrm{Z}_{12}\right)$ \\
\hline
\end{tabular}

(a)

\begin{tabular}{|l|l|l|l|l|}
\hline $\mathrm{I}\left(\mathrm{Y}_{20}\right)$ & $\mathrm{I}\left(\mathrm{Y}_{21}\right)$ & $\mathrm{I}\left(\mathrm{Y}_{22}\right)$ & $\mathrm{I}\left(\mathrm{Y}_{23}\right)$ & $\mathrm{I}\left(\mathrm{Y}_{24}\right)$ \\
\hline $\mathrm{I}\left(\mathrm{Y}_{19}\right)$ & $\mathrm{I}\left(\mathrm{Y}_{1}\right)$ & $\mathrm{I}\left(\mathrm{Y}_{2}\right)$ & $\mathrm{I}\left(\mathrm{Y}_{3}\right)$ & $\mathrm{I}\left(\mathrm{Y}_{9}\right)$ \\
\hline $\mathrm{I}\left(\mathrm{Y}_{18}\right)$ & $\mathrm{I}\left(\mathrm{Y}_{8}\right)$ & $\mathrm{I}\left(\mathrm{Y}_{0}\right)$ & $\mathrm{I}\left(\mathrm{Y}_{4}\right)$ & $\mathrm{I}\left(\mathrm{Y}_{10}\right)$ \\
\hline $\mathrm{I}\left(\mathrm{Y}_{17}\right)$ & $\mathrm{I}\left(\mathrm{Y}_{7}\right)$ & $\mathrm{I}\left(\mathrm{Y}_{6}\right)$ & $\mathrm{I}\left(\mathrm{Y}_{5}\right)$ & $\mathrm{I}\left(\mathrm{Y}_{11}\right)$ \\
\hline $\mathrm{I}\left(\mathrm{Y}_{16}\right)$ & $\mathrm{I}\left(\mathrm{Y}_{15}\right)$ & $\mathrm{I}\left(\mathrm{Y}_{14}\right)$ & $\mathrm{I}\left(\mathrm{Y}_{13}\right)$ & $\mathrm{I}\left(\mathrm{Y}_{12}\right)$ \\
\hline
\end{tabular}

(b)

Figure 4. (a) Sample image; (b) First order derivatives. 


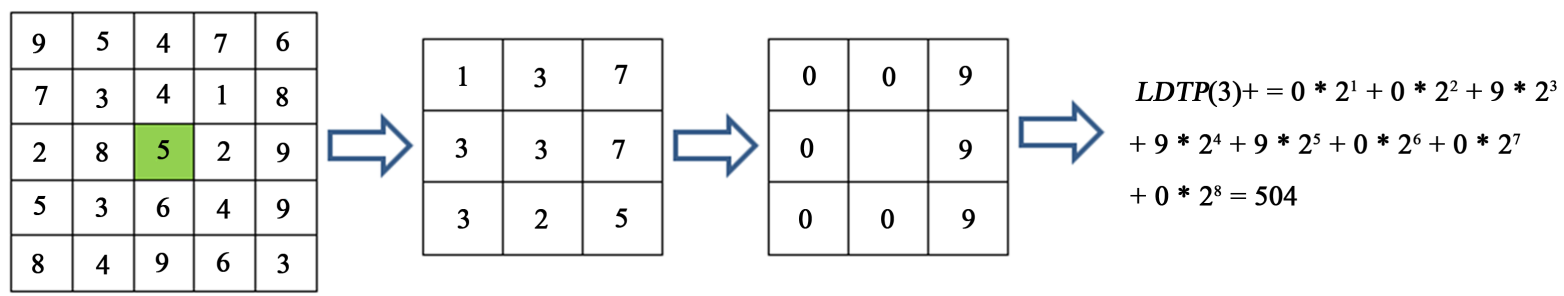

Figure 5. Computation of second order LDTP from a local region of size $5 \times 5$.

\subsection{Local Texture Description Framework Based Modified Local Directional Number Pattern}

Local Texture Description Framework based Modified Local Directional Number Pattern is a texture descriptor that deals with the directional information of a texture pattern that is formed with pixels at dissimilar areas in an image. Computation of LTDF_MLDN code comprises four stages:

* Detection of eight sampling points around a pixel along elliptical path using LTDF,

* Calculation of positive or negative response along each direction $i$ in a vector $\boldsymbol{v}$,

* Computation of the directional information of prominent positive and negative responses,

* Code determination. The detailed explanation of LTDF_MLDN is explained by Reena et al. [15].

\section{Experimental Result and Analysis}

\subsection{FKU Database}

PolyU finger knuckle print database [16] is used to evaluate the performance of the proposed method. This database is collected from 165 volunteers including 125 males and 40 females. These images are collected in two separate sessions. In each session, the subject is asked to provide six images for the left index and middle fingers, and the right index and middle fingers. In total, the database contains 7920 images with 12 images per finger. As images of a finger belong to a class, there are 660 classes. The database provides two sets of images. There are all original images in the first set, and the corresponding ROI images, with size of $110 \times 220$ pixels, are in another set. Some typical images are presented in Figure 6.

\subsection{Experimental Settings and Analysis}

\subsubsection{Performance Metrics}

In this paper, the performance metrics such as recognition rate, precision and recall are used for experimental investigation. True Positive Rate (TPR) which is based on True Positives (TP) and False Negatives (FN), False Positive Rate (FPR) based on False Positives (FP) and True Negatives (TN) and total number of tested samples (M) across all classes. The recognition rate is calculated based on True Positive Rate (recall) and False Positive Rate (precision) which is computed using the following Equations.

$$
\begin{aligned}
\text { Precision } & =\frac{\text { True Positive }}{\text { True Positive }+ \text { False Negative }} \\
\text { Recall } & =\frac{\text { True Positive }}{\text { True Positive }+ \text { False Positive }} \\
\text { Recognition Rate }(\%) & =\frac{\text { Number of images correctly classified }}{\text { Total number of images used }} * 100 .
\end{aligned}
$$

\subsubsection{Recognition Experiment Using All Fingers}

FKP images were randomly divided into training and testing set. 35\% of the total images are used for training and the rest for testing. Person-independent classification is done by representing the training set of images which was not included in the testing set of images. Each test was performed seven times and an average was calculated. In order to show the efficiency of multi resolution techniques, the experiments are conducted with and without preprocessing and the results are tabulated in Table 1. 


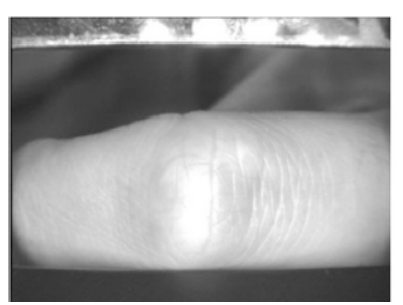

(a)

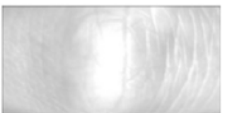

(c)

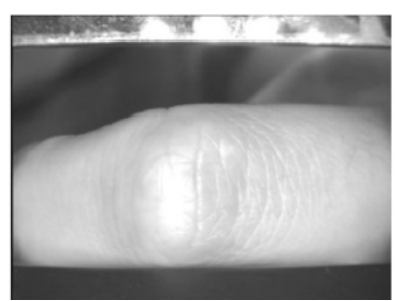

(b)

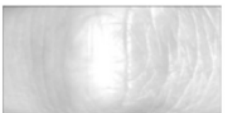

(d)

Figure 6. (a), (b) Original finger knuckle print images; (c), (d) Corresponding ROI images.

Table 1. Recognition rate of various texture models in multi resolution domain.

\begin{tabular}{ccccc}
\hline & \multicolumn{3}{c}{ Recognition Rate (\%) } \\
\cline { 2 - 5 } & \multicolumn{2}{c}{ Texture features only } & \multicolumn{2}{c}{ Texture features in Contourlet domain } \\
\cline { 2 - 5 } & NN classifier & ELM classifier & NN classifier & ELM classifier \\
LBP & 68 & 73.5 & 80 & 84 \\
LDN & 72 & 80 & 86 & 89 \\
LDTP & 80 & 88 & 94 & 97 \\
LTDF_MLDN & 78 & 85 & 90 & 98 \\
\hline
\end{tabular}

From Table 1, it is observed that, the LDTP features in Contourlet gives better recognition rate than other techniques. Contourlet transform detects the curves in more directions without being influenced by discontinuities. It also leads to extract smooth curves from images very effectively. The illumination variation does not affect all the parts equally. Hence, LDTP descriptor which is basically a local adaptive threshold-based method gives better result. One more experiment is conducted by varying the total number of testing images and precision, recall values are measured. The results are tabulated in Table 2.

From Table 2, it is observed that LDTP in Contourlet domain provides better results than the other models considered in this paper. The increase in the value of Precision and recall represents the better performance of the system.

\subsubsection{Recognition Experiment on Each Type of Finger}

In order to prove the efficiency of the proposed method, one more experiment is conducted to calculate the recognition rate of each finger and the EER results are tabulated in Table 3.

From Table 3, it is observed that, the proposed technique LDTP in Contourlet domain achieves much lower EER than the traditional methods in each type of finger. Specially, the left middle fingers perform better than the other fingers with the lowest EER of $0.15 \%$.

\subsubsection{Experiments about Computation Time}

In this experiment, the average processing time of all the images is measured and shown in Table 4. The total processing time of one image is less than $0.2 \mathrm{~s}$, which is fast enough for real-time applications.

From the above results, it is found that, computation time of LDTP is comparatively lower than the models considered in this paper.

\section{Conclusion and Future Scope}

LBP based FKP recognition under multiresolution based preprocessing is proposed in this paper. FKP is highly 
Table 2. FKP recognition using various texture models in multi resolution domain.

\begin{tabular}{ccccccc}
\hline \multirow{2}{*}{ Texture model } & \multicolumn{3}{c}{ Performance metric } \\
\cline { 2 - 7 } & Total images & TP & TN & FP & Precision & Recall \\
\hline LBP + Contourlet & 150 & 85 & 30 & 23 & 0.78 & 0.74 \\
LDN + Contourlet & 150 & 99 & 25 & 16 & 0.86 & 0.79 \\
LDTP + Contourlet & 150 & 140 & 4 & 2 & 0.98 & 0.97 \\
LTDF_MLDN + Contourlet & 150 & 140 & 5 & 3 & 0.97 & 0.96 \\
\hline
\end{tabular}

Table 3. EER (\%) of different fingers.

\begin{tabular}{ccccc}
\hline \multirow{2}{*}{ Method } & \multicolumn{3}{c}{ EER (\%) } \\
\cline { 2 - 5 } & Left index fingers & Left middle fingers & Right index fingers & Right middle fingers \\
\hline LBP + Contourlet & 7.50 & 7.31 & 6.87 & 7.04 \\
LDN + Contourlet & 1.47 & 0.74 & 1.23 & 1.42 \\
LDTP + Contourlet & 0.26 & 0.15 & 0.20 & 0.24 \\
LTDF_MLDN + Contourlet & 0.44 & 0.23 & 0.24 & 0.37 \\
\hline
\end{tabular}

Table 4. Recognition rate of various texture models in multiresolution domain.

\begin{tabular}{ccccc}
\hline \multirow{2}{*}{ Process } & \multicolumn{4}{c}{ Texture models in multiresolution domain } \\
\cline { 2 - 5 } & LBP + Contourlet & LDN + Contourlet & LDTP + Contourlet & LTDF_MLDN + Contourlet \\
\hline Feature extraction & 166.42 & 190 & 168.34 & 172 \\
Matching & 6.81 & 12 & 6.89 & 15 \\
Total & 173.23 & 202 & 175.23 & 187 \\
\hline
\end{tabular}

discriminable and unique which makes it an emerging promising biometric identifier. The proposed FKP technique is evaluated using database of all categories of Finger Knuckle samples. The method for human FKP images was implemented with NN and ELM classifier. Experimental results have demonstrated that LDTP is more feasible for FKP identification. LDTP and LTDF_MLDN in Contourlet domain achieve maximum accuracy of recognition rate of $98 \%$ with ELM classifier. FKP recognition depends on both feature extraction and classification algorithm. Hence, in future, the same problem can be experimented with different distance metrics in order to identify the suitable distance metric for recognition. FKP can be combined with other biometrics to propose a multimodal biometric system.

\section{References}

[1] (2003) Hand-Based Biometrics. Biometric Technology Today, 11, 9-11. http://dx.doi.org/10.1016/S0969-4765(03)07018-8

[2] Bolle, R., Pankanti, S. and Jain, A.K. (2000) Biometrics: The Future of Identification. Computer, 33, 46-49. http://dx.doi.org/10.1109/2.820038

[3] Zhang, L., Zhang, L. and Zhang, D. (2009) Finger-Knuckle-Print Verification Based on Band-Limited Phase-Only Correlation. 13th International Conference on Computer Analysis of Images and Patterns, Münster, Germany, 2-4 September 2009, 141-148. http://dx.doi.org/10.1007/978-3-642-03767-2_17

[4] Zhang, L., Zhang, L., Zhang, D. and Zhu, H. (2010) Online Finger-Knuckle-Print Verification for Personal Authentication. Pattern Recognition, 43, 2560-2571. http://dx.doi.org/10.1016/j.patcog.2010.01.020

[5] Aoyama, S., Ito, K. and Aok, T. (2011) Finger-Knuckle-Print Recognition Using BLPOC-Based Local Block Matching. The First Asian Conference on Pattern Recognition, Beijing, China, 28-30 November 2011, 525-529. 
http://dx.doi.org/10.1109/ACPR.2011.6166641

[6] Morales, A., Travieso, C., Ferrer, M. and Alonso, J. (2011) Improved Finger-Knuckle-Print Authentication Based on Orientation Enhancement. Electronics Letters, 47, 380-381. http://dx.doi.org/10.1049/el.2011.0156

[7] Badrinath, G., Nigam, A. and Gupta, P. (2011) An Efficient Finger-Knuckle-Print Based Recognition System Fusing Sift and Surf Matching Scores. The 13th International Conference on Information and Communications Security, Beijing, China, 23-26 November 2011, 374-387. http://dx.doi.org/10.1007/978-3-642-25243-3 30

[8] Zhang, L. and Li, H. (2012) Encoding Local Image Patterns Using Riesz Transforms: With Applications to Palm Print and Finger-Knuckle-Print Recognition. Image and Vision Computing, 30, 1043-1051. http://dx.doi.org/10.1016/j.imavis.2012.09.003

[9] Ekenel, H.K. and Sankur, B. (2005) Multiresolution Face Recognition. Image and Vision Computing, 23, 469-477. http://dx.doi.org/10.1016/j.imavis.2004.09.002

[10] Meena, K., Suruliandi, A. and Reena Rose, R. (2015) Enhancing the Performance of Texture-Based Face Recognition through Multi-Resolution Techniques. International Journal of Biometrics, 6, 363-386. http://dx.doi.org/10.1504/IJBM.2014.067132

[11] Do, M.N. and Vetterli, M. (2005) Thecontourlet Transform: An Efficient Directional Multiresolution Image Representation. IEEE Transactions on Image Processing, 14, 2091-2106. http://dx.doi.org/10.1109/TIP.2005.859376

[12] Ojala, T., Pietikäinen, M. and Maenpaa, T. (2002) Multiresolution Gray-Scale and Rotation Invariant Texture Classification with Local Binary Patterns. IEEE Transaction on Pattern Analysis and Machine Intelligence, 24, 971-987. http://dx.doi.org/10.1109/TPAMI.2002.1017623

[13] Rivera, A.R., Castillo, J.R. and Chae, O. (2013) Local Directional Number Pattern for Face Analysis: Face and Expression Recognition. IEEE Transactions on Image Processing, 22, 1740-1752. http://dx.doi.org/10.1109/TIP.2012.2235848

[14] Meena, K, Suruliandi, A. and Reena Rose, R. (2014) Face Recognition Based on Local Derivative Ternary Pattern. IETE Journal of Research, 60, 20-32. http://dx.doi.org/10.1080/03772063.2014.890811

[15] Reena Rose, R., Suruliandi, A. and Meena, K. (2015) Local Texture Description Framework Based Modified Local Directional Number Pattern: A New Descriptor for Face Recognition. International Journal of Biometrics, 7, 147-169. http://dx.doi.org/10.1504/IJBM.2015.070928

[16] The Hong Kong Polytechnic University (PolyU) Finger-Knuckle-Print Database. http://www4.comp.polyu.edu.hk/ biometrics/FKP.htm

\section{Submit or recommend next manuscript to SCIRP and we will provide best service for you:}

Accepting pre-submission inquiries through Email, Facebook, LinkedIn, Twitter, etc.

A wide selection of journals (inclusive of 9 subjects, more than 200 journals)

Providing 24-hour high-quality service

User-friendly online submission system

Fair and swift peer-review system

Efficient typesetting and proofreading procedure

Display of the result of downloads and visits, as well as the number of cited articles

Maximum dissemination of your research work

Submit your manuscript at: http://papersubmission.scirp.org/ 